\title{
A Multipronged Microscopy Approach Identifies Common Anti-Arrhythmic Strategy for Atrial Fibrillation and Myocardial Infarction
}

\author{
Louisa Mezache $^{1}$, Gerard Nuovo ${ }^{2}$ and Rengasayee Veeraraghavan ${ }^{1}$ \\ ${ }^{1}$ The Ohio State University, United States, ${ }^{2}$ Discovery Life Sciences, United States
}

Vascular leak and inflammation are associated with arrhythmic heart diseases such as atrial fibrillation (AF) and myocardial infarction (MI). AF is the most common form of arrhythmia, where the atria beat irregularly and rapidly. AF and other arrhythmias are a common complication following acute MI [1]. Despite its high prevalence and poor outcomes, the mechanism underlying AF is not well understood and treatment protocols for post-MI AF are limited.

AF patients have elevated levels of vascular endothelial growth factor (VEGF-A; 90-580 pg/ml), which promotes vascular leak and edema [2-4]. We investigated the structural underpinnings of AF using a novel approach we developed called indirect correlative light and electron microscopy (iCLEM). By exploiting structural landmarks identifiable via both light and electron microscopy, we were able to correlate transmission electron microscopy (TEM) -derived cardiac myocyte ultrastructure with molecular organization, assessed using sub-diffraction confocal imaging (sDCI) and stochastic optical reconstruction microscopy (STORM). Using this approach, we recently demonstrated that the vascular leak-inducing cytokine vascular endothelial growth factor (VEGF; $90-580 \mathrm{pg} / \mathrm{ml}$ - levels found in AF patients) induces acute remodeling (30-60 minutes) of sodium channel (NaV1.5) -rich intercalated disk (ID) nanodomains, disrupting their ultrastructure and prompting translocation of NaV1.5 from these sites. This in turn disrupted atrial impulse propagation (high speed optical mapping) and promoted atrial arrhythmias (electrocardiography). Here, we tested the hypotheses that i) similar acute pro-arrhythmic remodeling occurs in the ventricles of MI patients, and ii) protecting the vascular barrier may prevent arrhythmias following an acute inflammatory insult.

First, to determine cross-species similarities, we examined myocardial samples from five human MI patients by immunohistochemistry (IHC). VEGF was overexpressed in both cardiomyocytes and vascular endothelium in the border zone surrounding $<6$ month-old infarcts. Notably, co-localization analysis showed significantly reduced $\mathrm{NaV} 1.5$ near both connexin 43 and N-cadherin within the border zone, paralleling our observations in mouse atria. Next, we returned to our murine model of AF induced by acute inflammatory insult (100 pg/ml VEGF for 60 minutes) to test the antiarrhythmic efficacy of protecting the vascular endothelial barrier. We identified two molecular targets for vascular barrier protection, connexin43 (Cx43) and pannexin-1 (Panx1) [5,6]. In vascular endothelial cells, the opening of connexin43 hemichannels and pannexin channels, providing electrical and chemical coupling between cells and extracellular space, has been implicated in cytokine-induced vascular leak. Median in vivo arrhythmia burden was higher in VEGF-treated mice relative to vehicle controls $(7.5 \pm 11 \mathrm{vs}$. $0 \pm 6 \mathrm{~s} / \mathrm{hr})$. Blocking connexin43 hemichannels ( $\alpha$ CT11 peptide) decreased in vivo arrhythmia burden to $0 \pm 6.07$ s/hr. Panx1-IL2 (a peptide inhibitor of Panx1 channels) treatment also decreased in vivo arrhythmia burden $(0 \pm 15.57 \mathrm{~s} / \mathrm{hr}$ with 1.6 $\mu \mathrm{M}$ Panx1-IL2). Similar antiarrhythmic efficacy was also achieved with small molecule inhibitors of Cx43 and Panx1. 
Our multi-pronged microscopy approach allowed us to identify VEGF-induced vascular leak as a novel mechanism for acute arrhythmias and pinpoint the vascular barrier as a novel therapeutic target for both early stage AF and following MI.

\section{References}

[1] F Violi et al., JAHA 5 (2016), e003347.

[2] NA Chung et al., Stroke 33 (2002), p. 2187.

[3] J Li et al., Heart rhythm: The Official Journal of the Heart Rhythm Society 7 (2010), p. 438.

[4] Y Seko et al., Jpn Heart J 41 (2000), p. 27.

[5] J Jiang et al., JAHA 8 (2019), e012385.

[6] M Billaud et al., Sci Signal 8 (2015), p.364. 